\title{
Finite element models for laminated glass units with viscoelastic interlayer for dynamic analysis
}

\author{
T. Janda, A. Zemanová, J. Zeman \& M. Šejnoha \\ Faculty of Civil Engineering, Czech Technical University in Prague, \\ Czech Republic
}

\begin{abstract}
In this contribution, a methodology for calibration and validation of a finite element model for dynamic response of laminated glass units with viscoelastic interlayer is proposed. The model is based on a refined theory in which the adjacent layers are connected by kinematic constrains ensuring the inter-layer compatibility. The time-dependent behavior of the interlayer is accounted for by the generalized Maxwell model. The resulting system is solved by the Newton method with consistent linearization and allows for quantification of natural frequencies and eigenshapes. The calibration of the generalized Maxwell chain exploits the experimental master curve obtained from the rheometer test while the analysis of the dynamic response of glass beam serves for model validation.

Keywords: laminated glass, viscoelasticity, generalized Maxwell model, rheometer test, finite element method, natural frequency, dynamic analysis.
\end{abstract}

\section{Introduction}

Beside its traditional use in form of infill panes, glass gained popularity also as structural material in the past decades. This was possible mainly due to the invention of laminated glass consisting of glass layers connected with one or more polymer interlayers. The requirement for the glass components to bear external loading called for better understanding of the mechanical response under static, dynamic and impact loading. For safety reasons predicting the response of the glass units with one or more glass layers already fractured should also be possible.

In this paper we focus on a viscoelastic model of a three-layer laminated glass for dynamic analysis with emphasis on the development of methodology for its calibration based on rheometer measurements. Attention is limited to a model 




Figure 1: Generalized Maxwell model of viscoelastic material.

of naturally vibrating beam. This simple model will serve for validation of the proposed concepts and was chosen because the corresponding laboratory test is quite straightforward to perform. Details can be found in [1,2].

In this contribution we assume the laminated glass to be composed of two layers of strengthen glass and a single polymer interlayer. As usual, glass is described as linear elastic by the Young modulus $E$ and the Poisson ratio $\nu$. The behavior of the polymer interlayer is assumed viscoelastic and its mechanical properties are specified by the generalized Maxwell chain.

Lacking at present the experimental results, the paper concentrates on the theoretical grounds of the proposed model while verification against experimental data will be presented elsewhere. It is divided into two parts. The first part discuses the methodology for calibrating the parameters of the generalized Maxwell chain characterizing viscoelastic properties of the polymer interlayer based on experimental master curve obtained from a rheometer test. The second part formulates a finite element model of a glass beam with polymer interlayer suitable for a dynamic analysis and numerical determination of natural frequencies and modal shapes.

\section{Viscoelastic material}

In this section the complex viscoelastic shear modulus of the generalized Maxwell chain is derived. A stress-strain relationship described by the generalized Maxwell chain can be visualized as a parallel connection of single spring with stiffness $G_{\infty}$ and several Maxwell cells indexed by $i=1 \ldots n$, see Fig. 1 . The Maxwell cell itself is a serial connection of a spring of stiffness $G_{i}$ and a purely viscous damper of viscosity $\eta_{i}$.

For harmonic loading the stress and strain are defined in terms of complex values $\sigma^{*}$ and $\varepsilon^{*}$ and their relationship is given by

$$
\sigma^{*}=G^{*}(\omega) \varepsilon^{*}
$$

where

$$
G^{*}(\omega)=G^{\prime}(\omega)+\mathrm{i} G^{\prime \prime}(\omega),
$$


is the complex dynamic shear modulus, $\mathrm{i}=\sqrt{-1}$ is the imaginary unit and

$$
\begin{aligned}
& G^{\prime}(\omega)=G_{\infty}+\sum_{i=1}^{n} G_{i}^{\prime}(\omega), \\
& G^{\prime \prime}(\omega)=\sum_{i=1}^{n} G_{i}^{\prime \prime}(\omega)
\end{aligned}
$$

are the storage and loss moduli of the Maxwell chain, respectively. The quantities $G_{i}^{\prime}$ and $G_{i}^{\prime \prime}$ are the storage and the loss shear moduli of the $i$ th Maxwell cell which depend on the angual frequency $\omega$ and the parameters of the cell

$$
G_{i}^{\prime}(\omega)=\frac{\tau_{i}^{2} \omega^{2} G_{i}}{1+\tau_{i}^{2} \omega^{2}}, \quad G_{i}^{\prime \prime}(\omega)=\frac{\tau_{i} \omega G_{i}}{1+\tau_{i}^{2} \omega^{2}}
$$

where the characteristic time $\tau_{i}$ of the $i$ th Maxwell cell reads

$$
\tau_{i}=\frac{\eta_{i}}{G_{i}}
$$

This form is also referred to as the Prony series representation of the dynamic modulus.

\section{Calibration of the generalized Maxwell chain}

The parameters of the generalized Maxwell chain can be calibrated using the data from a rheometer test. The data consist of pairs of storage and loss moduli obtained for the selected angle of frequency sweep and particular temperature. These results then can be used to construct master curves for individual moduli [3]. Here, we shall consider the master curve as a collection of finite number of experimentally derived storage and loss moduli $\bar{G}_{j}^{\prime}$ and $\bar{G}_{j}^{\prime \prime}$ for a particular set of angular frequency $\omega_{j}, j=1 \ldots m$. The task now is to determine the shear moduli $G_{i}$ in the $i$ th cell of the generalized Maxwell chain while assuming fixed values of characteristic relaxation times $\tau_{i}$ such that these are reasonably distributed in the frequency band of interest. Formally, we want to minimize

$$
F\left(\left\langle G_{i}\right\rangle, G_{\infty}\right)=\sum_{j=1}^{m}\left(G^{\prime}\left(\omega_{j}\right)-\bar{G}_{j}^{\prime}\right)^{2}+\sum_{j=1}^{m}\left(G^{\prime \prime}\left(\omega_{j}\right)-\bar{G}_{j}^{\prime \prime}\right)^{2},
$$


by setting

$$
\begin{aligned}
\frac{\partial F}{\partial G_{i}} & =\sum_{j=1}^{m} 2\left(G^{\prime}\left(\omega_{j}\right)-\bar{G}_{j}^{\prime}\right) \frac{\tau_{i}^{2} \omega_{j}^{2}}{1+\tau_{i}^{2} \omega_{j}^{2}} \\
& +\sum_{j=1}^{m} 2\left(G^{\prime \prime}\left(\omega_{j}\right)-\bar{G}_{j}^{\prime \prime}\right) \frac{\tau_{i} \omega_{j}}{1+\tau_{i}^{2} \omega_{j}^{2}}=0, \\
\frac{\partial F}{\partial G_{\infty}} & =\sum_{j=1}^{m} 2\left(G^{\prime}\left(\omega_{j}\right)-\bar{G}_{j}^{\prime}\right)=0 .
\end{aligned}
$$

Taking into account equations (3) and (4) we can formulate the above conditions as a system of $n+1$ linear equations written as

$$
A x=b,
$$

with $n+1$ unknown optimal shear moduli stored in the column vector

$$
x=\left\{G_{1}, G_{2}, \ldots, G_{n}, G_{\infty}\right\}^{T} .
$$

The components of the matrix $A$ and the right hand side vector $b$ are provided by

$$
\begin{aligned}
A_{i j} & =\sum_{k=1}^{m} \frac{\tau_{i}^{2} \omega_{k}^{2}}{1+\tau_{i}^{2} \omega_{k}^{2}} \frac{\tau_{j}^{2} \omega_{k}^{2}}{1+\tau_{j}^{2} \omega_{k}^{2}}+\sum_{k=1}^{m} \frac{\tau_{i} \omega_{k}}{1+\tau_{i}^{2} \omega_{k}^{2}} \frac{\tau_{j} \omega_{k}}{1+\tau_{j}^{2} \omega_{k}^{2}} \\
A_{i n+1}=A_{n+1 i} & =\sum_{k=1}^{m} \frac{\tau_{i}^{2} \omega_{k}^{2}}{1+\tau_{i}^{2} \omega_{k}^{2}} \\
A_{n+1 n+1} & =m \\
b_{i} & =\sum_{k=1}^{m} \frac{\tau_{i}^{2} \omega_{k}^{2}}{1+\tau_{i}^{2} \omega_{k}^{2}} \bar{G}_{k}^{\prime}+\sum_{k=1}^{m} \frac{\tau_{i} \omega_{k}}{1+\tau_{i}^{2} \omega_{k}^{2}} \bar{G}_{k}^{\prime \prime} \\
b_{n+1} & =\sum_{k=1}^{m} \bar{G}_{k}^{\prime}
\end{aligned}
$$

where the $i, j=1 \ldots n$.

\section{Finite element model of the three layer beam}

Having derived the parameters of the generalized Maxwell chain together with the expression for the complex dynamic modulus we can start formulating the finite element model of a vibrating beam. Assuming the nodal displacements arranged in a column vector $r_{e}=\left\{u_{1}, w_{1}, \varphi_{1}, u_{2}, w_{2}, \varphi_{2}\right\}^{T}$ the stiffness matrix of a single 


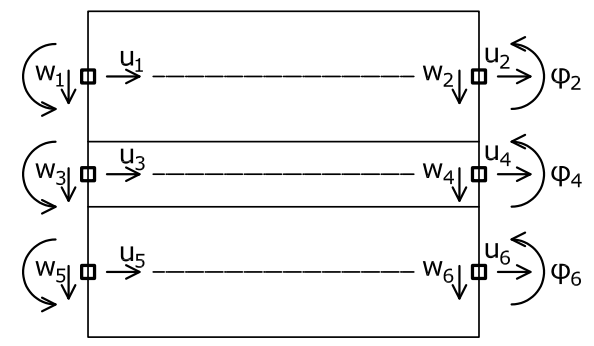

Figure 2: Composition of beam elements into super-element representing the three-layer material.

layer beam based on Mindlin's theory is written as

$$
K_{e}=\left[\begin{array}{cccccc}
\frac{E A}{L} & 0 & 0 & -\frac{E A}{L} & 0 & 0 \\
0 & \frac{G A^{*}}{L} & -\frac{G A^{*}}{2} & 0 & -\frac{G A^{*}}{L} & -\frac{G A^{*}}{2} \\
0 & -\frac{G A^{*}}{2} & \frac{G A^{*} L}{4}+\frac{E I}{L} & 0 & \frac{G A^{*}}{2} & \frac{G A^{*} L}{4}-\frac{E I}{L} \\
-\frac{E A}{L} & 0 & 0 & \frac{E A}{L} & 0 & 0 \\
0 & -\frac{G A^{*}}{L} & \frac{G A^{*}}{2} & 0 & \frac{G A^{*}}{L} & \frac{G A^{*}}{2} \\
0 & -\frac{G A^{*}}{2} & \frac{G A^{*} L}{4}-\frac{E I}{L} & 0 & \frac{G A^{*}}{2} & \frac{G A^{*} L}{4}+\frac{E I}{L}
\end{array}\right],
$$

where $E$ and $G$ are the Young and shear moduli, $A=b h$ is the cross section area, $A^{*}=\frac{5}{6} A$ is the effective area of the rectangular cross section, $I=\frac{1}{12} b h^{3}$ is the areal moment of inertia and $L$ is the element length. The element mass matrix reads

$$
M_{e}=\left[\begin{array}{cccccc}
\frac{\rho A L}{3} & 0 & 0 & \frac{\rho A L}{6} & 0 & 0 \\
0 & \frac{\rho A L}{3} & 0 & 0 & \frac{\rho A L}{6} & 0 \\
0 & 0 & \frac{\rho I L}{3} & 0 & 0 & \frac{\rho I L}{6} \\
\frac{\rho A L}{6} & 0 & 0 & \frac{\rho A L}{3} & 0 & 0 \\
0 & \frac{\rho A L}{6} & 0 & 0 & \frac{\rho A L}{3} & 0 \\
0 & 0 & \frac{\rho I L}{6} & 0 & 0 & \frac{\rho I L}{3}
\end{array}\right]
$$

where $\rho$ is the density of the material. Considering the layer composition of the super-element with nodes 1,3 and 5 on the left end and nodes 2, 4 and 6 on the 
right end, see Fig. 2, the stiffness matrix and the mass matrix become

$$
\begin{gathered}
K_{s e}=\left[\begin{array}{ccc}
K_{e, 1} & 0 & 0 \\
0 & K_{e, 2} & 0 \\
0 & 0 & K_{e, 3}
\end{array}\right], \\
M_{s e}=\left[\begin{array}{ccc}
M_{e, 1} & 0 & 0 \\
0 & M_{e, 2} & 0 \\
0 & 0 & M_{e, 3}
\end{array}\right] .
\end{gathered}
$$

Although using Lagrange multipliers to account for the connection between adjacent layers is more flexible, see [4], we introduce stiff kinematic constraints for their simplicity. The relative vertical displacements of the layers are constrained by

$$
\begin{aligned}
& w_{1}=w_{3}=w_{5}, \\
& w_{2}=w_{4}=w_{6} .
\end{aligned}
$$

We also assume a zero slip on the interfaces between the layers by setting

$$
\begin{aligned}
\varphi_{1} \frac{h_{1}}{2}+u_{1} & =-\varphi_{3} \frac{h_{2}}{2}+u_{3}, \\
\varphi_{3} \frac{h_{2}}{2}+u_{3} & =-\varphi_{5} \frac{h_{3}}{2}+u_{5}, \\
\varphi_{2} \frac{h_{1}}{2}+u_{2} & =-\varphi_{4} \frac{h_{2}}{2}+u_{4}, \\
\varphi_{4} \frac{h_{2}}{2}+u_{4} & =-\varphi_{6} \frac{h_{3}}{2}+u_{6} .
\end{aligned}
$$

Finally, choosing the master degrees of freedom

$$
r_{m}=\left\{u_{1}, w_{1}, \varphi_{1}, u_{5}, \varphi_{5}, u_{2}, w_{2}, \varphi_{2}, u_{6}, \varphi_{6}\right\}^{T},
$$

the reduced element stiffness matrix can be expressed as

$$
K_{r e}=T^{T} K_{s e} T .
$$

The transformation matrix $T$ derived from the constraints (21)-(26) is listed in the Appendix.

\section{Dynamic analysis}

The dynamic analysis of a vibrating beam starts from formulating the equation of motion

$$
K_{g}(\omega) r_{g}(t)+M_{g} \ddot{r}_{g}(t)=0,
$$

where $K_{g}$ and $M_{g}$ are the global stiffness and mass matrices obtained from the reduced element matrices $K_{r e}$ and $M_{r e}$ by localization. For harmonic oscillations 
we assume the displacement and rotations in the form

$$
r_{g}(t)=e^{i \omega t} r_{g}
$$

Their second derivatives, i.e. accelerations thus become

$$
\ddot{r}_{g}(t)=-\omega^{2} e^{i \omega t} r_{g}
$$

The equation of motion then rewrites as

$$
\left(K_{g}(\omega)-\omega^{2} M_{g}\right) r_{g}=0
$$

Any non-zero solution $r_{g}$ of this system can occur only for angular frequencies $\omega$ which satisfy

$$
\operatorname{det}\left(K_{g}(\omega)-\omega^{2} M_{g}\right)=0
$$

Note that, due to the complex stiffness modulus in the element with viscoelastic layer, the stiffness matrix is complex-valued quantity and depends additionally on the angular frequency.

For the middle layer, which is assumed to behave viscoelastically, we split the complex shear modulus into the constant real part $G_{\infty}$ and the complex frequency dependent part $G_{\omega}$

$$
G^{*}(\omega)=G_{\infty}+G_{\omega}(\omega)
$$

This also allows us to separate the real-valued, $\omega$-independent part of the global stiffness matrix from the complex-valued and $\omega$-dependent one. Assuming $E_{\omega}(\omega)=2(1+\nu) G_{\omega}(\omega)$ we write

$$
K_{g}(\omega)=K_{g, \infty}+G_{\omega}(\omega) K_{g, \omega}
$$

The strategy for the solution of viscoelastic beam is described in [5] and employs the Newton-Raphson method with initial values being the natural frequency and the eigenshape of the chosen mode obtained for a purely elastic beam. Thus in the first step we find the natural frequencies and eigenshapes for the beam with the real $\omega$-independent part of the global stiffness matrix $K_{g, \infty}$, i.e. we search for nonzero solutions of

$$
\operatorname{det}\left(K_{g, \infty}-\omega^{2} M_{g}\right) r_{g}=0
$$

which represents the generalized eigenvalue problem for pair $\left(K_{g, \infty} ; M_{g}\right)$. The obtained eigenvalues correspond to squares of natural angular frequencies and the eigenvectors correspond to shapes in which the beam vibrates with these frequencies. 
The Newton-Raphson method is applied next. Recall, that the method searches for a vector $x$ such that the vector valued function $f$ satisfies

$$
f(x)=0 .
$$

This is achieved by iterating

$$
x_{k+1}=x_{k}-J^{-1}\left(x_{k}\right) f\left(x_{k}\right) .
$$

In our application the vector of unknowns consists of components of $r_{g}$ and the angular frequency $\omega$ :

$$
x=\left\{r_{g}, \omega\right\}^{T} .
$$

Its initial values $r_{g 0}$ and $\omega_{0}$ are obtained from the eigenvalue decomposition as disused above. The components of function $f(x)$ consists of the left hand side expressions of the equation of motion (33) regularized by the requirement of orthogonality between the initial eigenshape and its increment

$$
\begin{aligned}
f_{i}(x) & =\sum_{j=1}^{n}\left(K_{g, i j}(\omega)-\omega^{2} M_{g, i j}\right) r_{g, j}, \\
f_{n+1}(x) & =r_{g 0}^{T}\left(r_{g}-r_{g 0}\right) .
\end{aligned}
$$

The components of the Jacobian matrix $J(x)$ are defined

$$
\begin{aligned}
J_{i j}(x) & =K_{g, i j}(\omega)-\omega^{2} M_{g, i j}, \\
J_{i n+1}(x) & =\sum_{j=1}^{n}\left(\frac{\mathrm{d} G_{\omega}(\omega)}{\mathrm{d} \omega} K_{g, \omega, i j}-2 \omega M_{g, i j}\right) r_{g, j}, \\
J_{n+1 j}(x) & =r_{g 0, j}, \\
J_{n+1 n+1}(x) & =0
\end{aligned}
$$

where

$$
\frac{\mathrm{d} G_{\omega}}{\mathrm{d} \omega}=-\sum_{j=1}^{n} \frac{\mathrm{i} \tau_{j} G_{j}}{\left(\tau_{j} \omega_{j}-\mathrm{i}\right)^{2}}
$$

with i being the imaginary unit. Note, that the components of the vector $x$, although starting from real initial values, become complex numbers after the first iteration. If desired this procedure can be repeated for initial frequency found from (36).

\section{Conclusions}

This paper presented a numerical strategy for calibration of the parameters defining the mechanical properties of the viscoelastic interlayer connecting two glass panes 
into laminated glass unit. The viscoelastic material is described by the generalized Maxwell model which yields a complex dynamic modulus in a form that is suitable for finite element model of a laminated beam under dynamic excitation. The analysis of natural harmonic vibrations requires solving the frequency-dependent eigenvalue problem in the complex domain. This is achieved by exploiting the Newton iterative method. The formulation of the finite element model of naturally vibrating beam was motivated by accessibility of corresponding physical experiment against which the model of the layered element can be validated. This will be presented in our forthcoming paper.

\section{Acknowledgement}

The financial support provided by the GAČR grant No. GA16-14770S is gratefully acknowledged.

\section{References}

[1] Andreozzi, L., Briccoli Bati, S., Fagone, M., Ranocchiai, G. \& Zulli, F., Dynamic torsion tests to characterize the thermo-viscoelastic properties of polymeric interlayers for laminated glass. Construction and Building Materials, 65, pp. 1-13, 2014.

[2] Šejnoha, M. \& Zeman, J., Micromechanics in Practice. WIT Press, 2013.

[3] Williams, M., Landel, R. \& Ferry, J., The temperature dependence of relaxation mechanisms in amorphous polymers and other glass-forming liquids. Journal of the American Chemical Society, 77(14), pp. 3701-3707, 1955.

[4] Zemanová, A., Zeman, J. \& Šejnoha, M., Finite element model based on refined plate theories for laminated glass units. Latin American Journal of Solids and Structures, 15, pp. 1158-1180, 2015.

[5] Daya, E.M. \& Potier-Ferry, M., A numerical method for nonlinear eigenvalue problems application to vibrations of viscoelastic structures. Computers and Structures, 79, pp. 533-541, 2001.

\section{Appendix}

Transformation matrix maps all degrees of freedom in super element $r_{s}=$ $\left\{u_{1}, w_{1}, \varphi_{1}, u_{3}, w_{3}, \varphi_{3} u_{5}, w_{5}, \varphi_{5}, u_{2}, w_{2}, \varphi_{2} u_{4}, w_{4}, \varphi_{4}, u_{6}, w_{6}, \varphi_{6}\right\}^{T}$ to the master degrees of freedom $r_{m}=\left\{u_{1}, w_{1}, \varphi_{1}, u_{5}, \varphi_{5}, u_{2}, w_{2}, \varphi_{2}, u_{6}, \varphi_{6}\right\}^{T}$ of the reduced element. Taking into account the constraints introduced by 
Eqs. (21)-(26), this matrix can be expressed as:

$$
T=\left[\begin{array}{cccccccccc}
1 & 0 & 0 & 0 & 0 & 0 & 0 & 0 & 0 & 0 \\
0 & 1 & 0 & 0 & 0 & 0 & 0 & 0 & 0 & 0 \\
0 & 0 & 1 & 0 & 0 & 0 & 0 & 0 & 0 & 0 \\
0 & 0 & 0 & 0 & 0 & 1 & 0 & 0 & 0 & 0 \\
0 & 0 & 0 & 0 & 0 & 0 & 1 & 0 & 0 & 0 \\
0 & 0 & 0 & 0 & 0 & 0 & 0 & 1 & 0 & 0 \\
\frac{1}{2} & 0 & \frac{h_{1}}{4} & \frac{1}{2} & -\frac{h_{3}}{4} & 0 & 0 & 0 & 0 & 0 \\
0 & 1 & 0 & 0 & 0 & 0 & 0 & 0 & 0 & 0 \\
-\frac{1}{h_{2}} & 0 & -\frac{h_{1}}{2 h_{2}} & \frac{1}{h_{2}} & -\frac{h_{3}}{2 h_{2}} & 0 & 0 & 0 & 0 & 0 \\
0 & 0 & 0 & 0 & 0 & \frac{1}{2} & 0 & \frac{h_{1}}{4} & \frac{1}{2} & -\frac{h_{3}}{4} \\
0 & 0 & 0 & 0 & 0 & 0 & 1 & 0 & 0 & 0 \\
0 & 0 & 0 & 0 & 0 & -\frac{1}{h_{2}} & 0 & -\frac{h_{1}}{2 h_{2}} & \frac{1}{h_{2}} & -\frac{h_{3}}{2 h_{2}} \\
0 & 0 & 0 & 1 & 0 & 0 & 0 & 0 & 0 & 0 \\
0 & 1 & 0 & 0 & 0 & 0 & 0 & 0 & 0 & 0 \\
0 & 0 & 0 & 0 & 1 & 0 & 0 & 0 & 0 & 0 \\
0 & 0 & 0 & 0 & 0 & 0 & 0 & 0 & 1 & 0 \\
0 & 0 & 0 & 0 & 0 & 0 & 1 & 0 & 0 & 0 \\
0 & 0 & 0 & 0 & 0 & 0 & 0 & 0 & 0 & 1
\end{array}\right] .
$$

